\title{
On-the-Fly Situation Composition within Smart Spaces
}

\author{
Prem Prakash Jayaraman ${ }^{1}$, Arkady Zaslavsky ${ }^{2}$, Jerker Delsing², \\ ${ }^{1}$ Caulfield School of Information Technology, Monash University, \\ 900 Dandenong Road, Melbourne, Victoria 3145 \\ \{prem.jayaraman\} @ infotech.monash.edu.au.au \\ ${ }^{2}$ Department of Computer Science and Electrical Engineering, \\ Lulea University of Technology, Lulea, Sweden \\ \{arkady.zaslavsky, jerker.delsing\} @ ltu.se
}

\begin{abstract}
Advances in pervasive computing systems have made smart computing spaces a reality. These smart spaces are sources of large amount of data required for context aware pervasive applications to function autonomously. In this paper we present a situation aware reasoning system that composes situations at runtime based on available information from the smart spaces. Our proposed system R-CS uses situation composition on-the-fly to compute temporal situations that best represent the real world situation (contextual information). Our proposed situation composition algorithm is dependent on underlying sensor data (hardware and software). These sensory data are prone to errors like inaccuracy, old data, data ambiguity etc. R-CS proposes algorithms that incorporate sensor data errors estimation techniques into our proposed dynamic situation composition based reasoning system. R-CS is built as an extension to Context Spaces, a fixed situation set based reasoning system. We implement R-CS dynamic situation composition algorithms over context spaces and validate our proposed R-CS model against context spaces' fixed situation reasoning model.
\end{abstract}

Keywords: Context Modelling, Smart Spaces, Reasoning Under Uncertainty

\section{Introduction}

Pervasive systems depend on contextual information to perform tasks in smart environments. The advances in pervasive computing research have made Smart Spaces a possibility. A smart space is a smart environment that has commuting embedded into it and can provide information that can be used to model the real world into the virtual computing world. We use the term smart spaces to represent such environments in the context of this paper. This contextual information originates from individual or multiple sensory sources. The sensor data by itself has no meaning but when fused with appropriate context provides a virtual view of the physical Smart Space. We define "Context" as "that which surrounds, and gives meaning to, something else” [1]. In Smart Spaces (pervasive environments), our definition of Context is realized by fusing data obtained from multiple sensors within the environment. 
In this paper we use a situation based reasoning approach. A situation is a predefined collection of contextual elements and is inferred based on current environmental context. For e.g. in a smart room, based on sensor inputs for light, noise and location, we can infer the occurrence of a situations Meeting or Presentation. Hence a situation is a virtual world definition of a real world Smart Space. The situation based reasoning model Ranked-Context Spaces (R-CS) proposed in this paper evolves from Context Spaces (CS) [2, 14]. CS is a theoretical approach towards modelling context using situations. Each situation is a composition of context attributes with well defined regions. The CS model uses a fixed set definition of situations. This is acceptable in most cases but in Smart Spaces where new sensory inputs appear and disappear, the present CS system does not scale. We extend CS fixed situation space representation to a dynamic situation spaces composition where the situation space computed on the run is a collection of context attributes with it corresponding region that best defines the current situation within the smart space. To achieve this we use simple multi-attribute rating technique (SMART) [4] instead of the multi attribute utility theory (MAUT) [5] used by CS. The paper also proposes and implements algorithms into R-CS to incorporate sensor inaccuracies that improves the reasoning accuracy. By incorporating dynamic situation composition into CS model, our context model which is a multi dimensional virtual world representation of the physical smart space grows and shirks in with changing contextual attributes. We use the term multi-dimensional to represent multiple context attribute used to compose a situation.

The key contribution of the paper includes proposing and implementing R-CS which incorporates dynamic situation composition and a novel sensor inaccuracy estimation algorithm into CS. We argue that current error estimation and fixed situation set representation of smart spaces used by CS is insufficient for changing pervasive environments. R-CS aims to develop and propose solutions to these challenges identified in CS. This papers contribution is a further work to our previous work [15].

The rest of the paper is organized as follows. Section 2 presents related works in the area of context modelling. Section 3 provides a background into context spaces theory. Section 4 presents our proposed dynamic situation composition system R-CS, and our proposed sensor inaccuracy estimation algorithm which has been implemented in R-CS. Section 5 validates our proposed R-CS approaches by comparing the outcome of situation reasoning to CS approach. Section 6 concludes the paper.

\section{Context Reasoning: An Overview}

Reasoning technologies generally used in context-aware pervasive system include rule based logic, Bayesian network and neural network. Pervasive environments need models that can handle reasoning under degrees of context varying uncertainty. Context modelling and reasoning has been explored and presented by number of researchers. Context ToolKit [11] is a framework that presents a framework for 
interfacing between devices and software entities that provide contextual information. Five context abstractions namely Widgets, Interpreters, Aggregators, Services and Discoverers can be used by context-aware application developers to prototype applications. The widgets acquire context information from sensors, interpreter interprets that into high level information and aggregator collects related context information for a specific entity. Services execute behaviours and discovers maintain a list of services available to the application. Applications use discoverers to find specific components or a set of component that match certain criteria. Context models based on Logic and ontology have been presented in [12, 13]. In this paper we look at situation based reasoning approach. A situation is a high level context which is inferred from low level context obtained from individual sensors (context attributes). A situation comprises number of context attributes, each having a well defined region within the specific situation space. The major challenge in situation reasoning is reasoning under uncertainty. One of the methods used for reasoning under uncertainty is Bayesian reasoning. In [6], a Bayesian technique for location estimation based on inaccurate sensor values and [7] uses this approach for computing location of devices indoor. Applying Bayesian approach has the limitation of prior knowledge of probabilities which may not be always available. Fuzzy based systems [3] have been used for controlling systems based on Fuzzy Rule set. Though Fuzzy systems are useful in controlling system process, they are less effective in reasoning situations [8] especially when we have situations with overlapping regions which makes reasoning under uncertainty harder. Neural network based approaches for classifying context has several shortfalls when used with quickly deployable context aware applications. Neural network require an extensive training phase over sufficient number of patterns. Training can result in local minima, i.e., achieving less than optimal solutions [9]. Neural network are considered "black boxes" as it's difficult to back track problem to the cause.

Review of context modelling approaches show that most have shortfalls which makes it difficult to provide a generic approach for context modelling under changing situations and applications domains. To this extent most of the work in the literature has not addressed the problem of dynamic smart spaces where new input data may influence the reasoning process. CS is also one such context model which provides a generic framework for context aware application to reason based on situation modelling. As mentioned in previous section, CS still does not address changing smart spaces. In the next section we present an overview of Context Spaces (CS) theory and introduce the heuristics incorporated in CS. We then identify the shortfalls of CS and propose additional heuristics incorporated into R-CS to aid reasoning under uncertainty in Smart Spaces.

\section{Context Spaces}

The term context has a number of definitions. In general it refers to a particular centre of interest, providing added information about whom, where and when. We define context as a representation scheme for data that can be used to compose 
situations which can be reasoned upon. We use figure 1 o depict the relation between context and situations. The contextual information is dependent on underlying sensor data (software and hardware) whose relations are computed by adding context to them at the context layer. The Situations layer is a composition of related context attributes each having a well defined region that best describes a situation. (E.g. Meeting, Presentation). CS uses the following definitions to represent the context-situation pyramid in the CS model $[2,14]$.

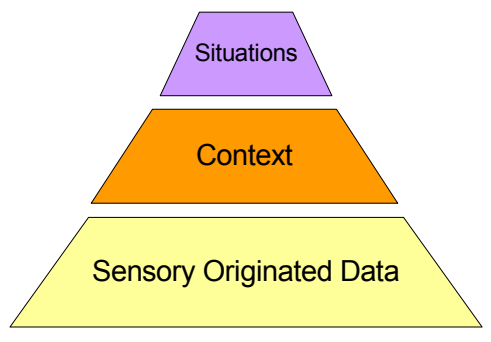

Figure 1: Context - Situation Pyramid
A "Context Attribute" defines data obtained from individual or group of sensor that is used in situation reasoning $[2,14]$. By itself a "Context Attribute" is a single piece of data that has no meaning until we relate context to it (E.g.: Temperature vs. Temperature of Human Body). A "Situation Space" is a collection of context attributes that define a situation. Each context attribute within the situation has a well defined region. A "region" of a context attribute is a set of acceptable values that falls within the specific situation definition. CS uses a crisp representation of "region". E.g. For a situation "Moderate Operating Environment of Machine A" context attribute temperature would be within the region " $25-28{ }^{\circ} \mathrm{C}$ ". "Context State" represents the current value of a context attribute. CS uses multi attribute utility theorem to compute confidence in situation occurrence [2, 14]. The confidence is the measurement parameter used to determine the occurrence of a situation. To compute the confidence, CS uses two primary heuristics

1. Inaccuracies of Sensors using error probability

2. Contributing and Relevance factor of each context attribute $a_{i}$ in the situation space $\mathrm{S}$.

The heuristics identified provides sensor error probability, relevance and contribution value of the context attribute. The relevance and contribution are values given to the context attributes based on it context state (current value) in the situation space S. A contribution function computes the contribution (between 0 and 1) of context attribute $\mathrm{a}_{\mathrm{i}}$ over the region Ai defined in the situation space $\mathrm{S}$. The relevance factor gives the attributes importance in the situation $S$. The situation space definition of CS has fixed set context attribute regions $\left(A_{1}, A_{2}, A_{n}\right)$ whose importance (relevance) is given by

$$
\sum_{i=0}^{n} w_{i}=1
$$

We argue that in real world smart environments, this may not always be the case. Hence we propose the use of an extended situation space set and compute a temporal situation space on the run at every instance t, from available set of context attribute regions. The probabilistic error computation used in CS needs to have pre-determined probability but does not learn from other information like sensor data freshness, noise etc. Our proposal incorporates data freshness to estimate the sensor in-accuracy in 
addition to the probabilistic approach employed by CS aiming to decrease sensor inaccuracy caused due to aging data.

\section{Situation Composition on-the-fly}

The dynamic situation composition arises from our argument that fixed situation based reasoning does not cater to changing smart spaces where new context information is appears and disappears. Hence we need a model that can adapt to changing situations and compute situation definitions dynamically using available context attributes. Our proposal is to compute situation dynamically based on changing real world situations. We extend the situation space presented in CS from a finite set of highly relevant attribute to a universal situation space of all possible (not infinity) attributes. At any given instance t, R-CS builds a situation space on the run based on sensory input available within the smart space that has a equivalent context attribute definition in the universal situation space. Since our situation space definition is an extended set of all possible attributes, we use simple multi-attribute rating technique (SMART) [4] instead of MAUT used in CS. SMART ranks the attributes regions based on relevance and contributing factor. We also employ data quality parameters like data freshness into R-CS which is important while reasoning applications based on sensor originated data. Since our temporal situation space is a subset of the universal situation space, our context model that best represents the smart spaces grows and shrinks in size (attributes).

\subsection{Situation Composition based on Smart Space Information}

In [2] CS Situation space is defined as, a representation of a real world situation. Our proposal is to define a generic universal situation space as $S_{U}$, a representation of situation with all possible combinations of context attributes and corresponding weights. As against the context spaces definition of weights in equation (1) we define this weight $w_{i}$ denoting the importance of the attribute region to the situation to be a value between a lower and a higher scale (application specific). CS [2, 14] uses MAUT approach to compute utility. We propose the use of (SMART) [4] that employs MAUT but adds ranking to MAUT. The ranking of a context region is dynamic and is dependent on both the relevance and it contribution to the situation. Based on this ranking, a temporal situation space $\mathrm{S}_{\mathrm{T}}$ is compute at runtime.

Definition1: Situation Space $\left(S_{U}\right)-A$ universal situation space which has all possible sets of context attributes and corresponding regions with their importance

$$
S_{U}=\left\{w_{1} A_{1}, w_{2} A_{2}, \cdots w_{n} A_{n}\right\} \text { where } \min \leq w \leq \max
$$


Definition2: Situation Space $\left(S_{T}\right)$ - A temporal situation space at time $T$ which represents the real world situation with delta changes that has a list of context attributes and regions that best defines the situation at time $T$.

$$
S_{T}=\left\{w_{1} A_{1}, w_{2} A_{2}, \ldots w_{m} A_{m}\right\} \text { where } m \leq n, \sum_{i=0}^{m} w_{i}=1 \text { and } S_{T} \subseteq S_{U}
$$

The temporal situation space $\mathrm{S}_{\mathrm{T}}$ is a dynamic representation of the situation at time $t$ and changes as situation evolves. The temporal situation space is composed of the highly ranked attributes which is determined by the context attributes contribution and relevance values. By the use of a dynamic temporal situation composition, R-CS enforces dynamic ranking of attributes at runtime hence moving away from fixed situation space definitions to situation space definitions dynamically.

The universal situation space at any point in time has $\mathrm{n}$ number of context attributes. Hence we propose a partition algorithm that partitions the situation space based on the relevance of the context attribute. This partition is application specific. The reason we chose a partitioned situation space is to reduce the complexity of iterating through all the attributes when confidence can be reached by considering the first few partitions.

\subsection{Partitioning Parameter $\delta$}

Relevance, the importance of the attribute region in the situation space $\mathrm{S}_{\mathrm{U}}$ is one of our key parameter to compute the partitioned situation space. We define a partition parameter $\delta$ called partitioner.

Definition 3: Partition $\delta$ is defined as set of conditions $C$ that satisfy a predicate $P$ i.e. $\{C \mid P(C)\}$.

The partition parameter partitions the universal situation space based on the predicate $\mathrm{P}$. We use this partitioned space to compute the temporal situation space which takes into consideration the attributes contribution. E.g. the contribution for context attribute temperature for the situation "Machine Operating GOOD" will be 1 if temperature has value between 17 and 19 and will be 0 if it is outside the region. The temporal situation space is computed as a function of the relevance (weight) and the contribution. The algorithm iterates through every partition of the universal situation space until a confidence threshold is reached. The confidence threshold is defined as the difference in the confidence of the situations being reasoned. The higher the threshold, higher is the certainty of occurrence of one situation over the other. To reduce weight distribution while situations overlap, we propose a weight redistribution algorithm. The situation overlap occurs when non-coexisting situations attribute regions overlap with each. The weight redistribution is performed over the temporal space hence preserving the weights of context regions in the universal situation space. While reasoning two situations which are non-coexistent, the weights (importance) associated with that attribute regions is recomputed using the equation 


$$
\frac{\operatorname{Max}\left(w_{i}\right)-\operatorname{Min}\left(w_{i}\right)}{\operatorname{Max}\left(w_{i}\right)+\operatorname{Min}\left(w_{i}\right)}
$$

where $\mathrm{w}_{\mathrm{i}}$ is the set of weights assigned to attribute regions in the temporal situation space $\mathrm{S}_{\mathrm{T}}$. Once the weight of overlapping attribute regions is computed, the entire situation space weights are normalized to compute the new weights. Figure 2 and 3 illustrates the pseudo-code to re-compute weights for overlapping regions and code to partition the Universal situation space.

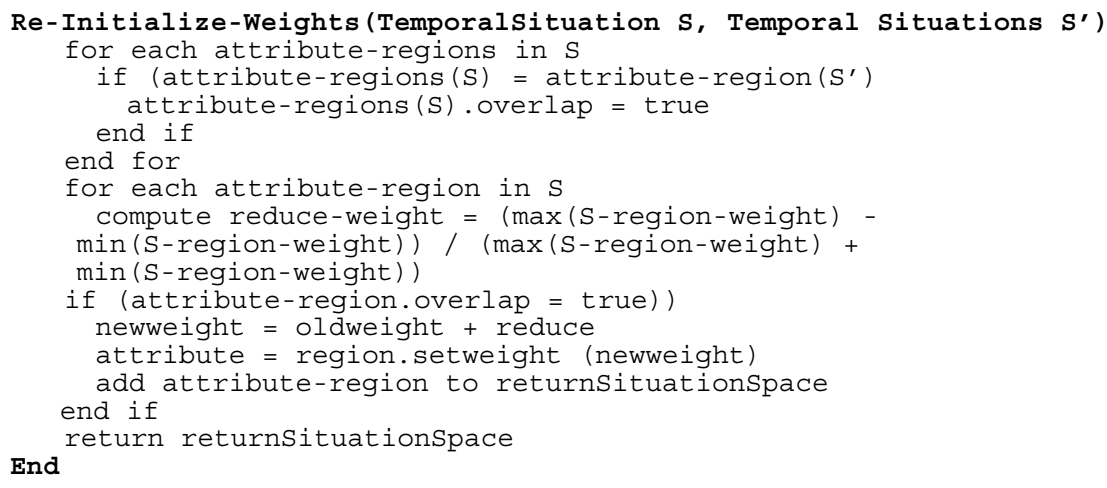

Figure 2: Pseudo Code to recomputed overlapping attribute regions weights

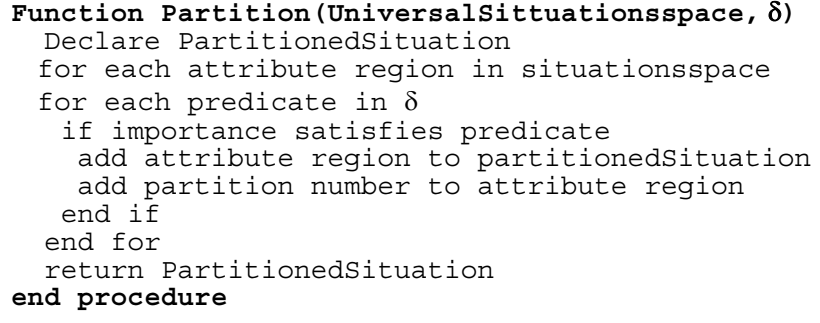

Figure 3: Pseudo Code to partition universal situation space $\mathrm{S}_{U}$

\subsection{Incorporating Sensor Inaccuracy into Dynamic Situations}

The sensor inaccuracy used by CS is both primitive and has crisp boundary. The contribution using the error function is computed using the formula 


$$
c_{i}=P_{\text {error }} * \text { RgionContribution }
$$

The RgionContribution is computed using a crisp boundary and is determined by checking if the sensor value falls within the attribute region. If it does not a value 0is returned and if it is a value 1 is returned. The $P_{\text {error }}$ provides the probability by which the sensor reading is accurate. The crisp region does not suit all real world cases. Also CS does not change the sensor value based on the error probability. E.g. if there is $5 \%$ error, then the value can have a \pm 0.05 variation from the outer boundaries. This is not considered in CS while computing the contribution. Hence we propose the use of non-crisp boundaries as illustrated in figure 4 .

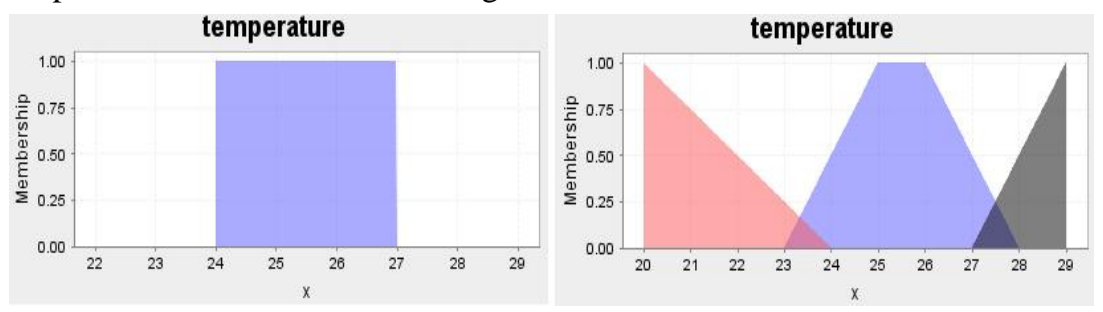

Figure 4: Comparison between CS Crisp boundary and E-CS membership

To convert the crisp region into a value based on bordering regions, we employ the principle used by Fuzzy membership functions [3]. E.g. if we define a situation HOT whose temperature can be ranging from 34 to 40 , a real value (context state) of 40.05 can still fall under the condition HOT if we have some tolerance in the outer regions of the context attribute. Our technique focuses on values that are inside and at the border of the defined region. Hence we re-define the context attribute's region with two values namely outer range and inner range. The inner range represents values which returns contribution factor of 1 while the outrange is a \pm value to the inner range values that returns a contribution based on where the sensor value falls in the outer range. Our approach tests the sensor value against the inner and outer region after applying the error probability over the sensed value. To compute sensor inaccuracy, we also introduce data freshness. By data freshness, we mean how recent the data is? We do not get into quality of context [10] as that is a topic of research by itself. Incorporating probabilistic sensed value and sensor data freshness, our new contribution function is given in (7). We implement these inaccuracy estimation algorithms into our proposed system. The freshness parameter is application specific.

$$
c_{i}=\text { RgionContribution }_{i} * \sigma_{i} \text { where }_{i}=P_{\text {error }} * \text { Freshness }
$$

$\sigma$ is the composite error computed from probability of error and freshness. To improve sensor accuracy, more data quality related parameters can be added to $\sigma$. 


\section{Simulation and Evaluations}

R-CS has been implemented over CS model for sensor fusion and reasoning by incorporating the proposed algorithms for situation partitioning, re-computation of weights and improved error estimation. We present our evaluation by validating the proposed approaches against CS approach.

\subsection{Sensor Inaccuracy}

\begin{tabular}{|lll|}
\hline Situation: & Presentation & \\
Attributes & Regions & \\
\hline LIGHT & ">-=10" & $"<=20 "$ \\
NOISE & $">20 "$ & $"<30 "$ \\
& & $=$ \\
PROJECTOR & $"=$ ON" & OFF" \\
PEOPLE & $">5 "$ & "< $10 "$ \\
\hline
\end{tabular}

Table 1: Presentation Situation Definition

We compare CS based confidence generation and R-CS based confidence generation using our proposed probabilistic incorporated, freshness based error computation algorithm. To test the effect of the improved error estimation algorithm, we ran simulations to reason a Presentation situation. The situation definition is shown in Table 1. Table 2, provides the output of using probabilistic error correction and data freshness technique. As it shows, the R-CS system computes a confidence of 1 for attribute noise taking into consideration its error rate while returns 0 to people attribute taking into consideration the freshness threshold is greater than 3 .

\begin{tabular}{|lllrrrr|}
\hline $\begin{array}{c}\text { Threshold } \\
>2\end{array}$ & Regions & $\begin{array}{c}\text { Actual } \\
\text { Sensor } \\
\text { Values }\end{array}$ & \multicolumn{1}{l}{ Error } & Freshness & $\begin{array}{l}\text { CS } \\
\text { Confidence }\end{array}$ & R-CS \\
\hline Light & 10 to 20 & 12 & $90 \%$ & 1 & 1 & 1 \\
Noise & 20 to 30 & 19.95 & $90 \%$ & 2 & 0 & 1 \\
People & 5 to 10 & 10 & $100 \%$ & 3 & 1 & 0 \\
\hline
\end{tabular}

Table 2: Comparing CS and R-CS error estimation approach

The final result of our simulation run is shown in figure 4. From the graph, we can see that variation in freshness and error probability reflects on the final confidence measure generated by R-CS while it has not effect on CS. At simulation run 3, the

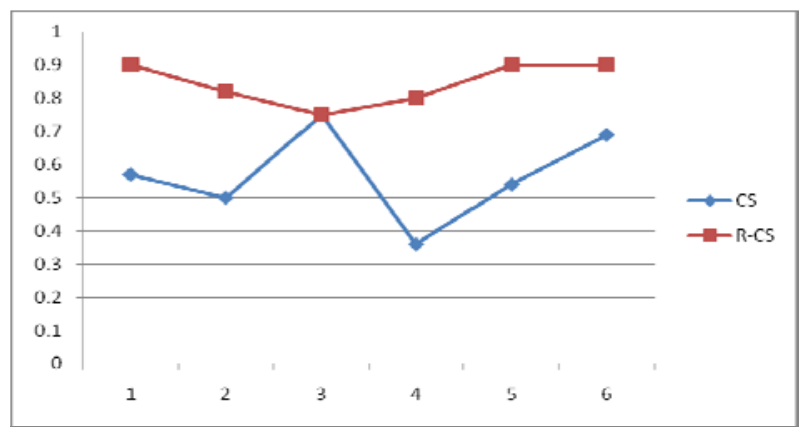

Figure 4: Varying Freshness Threshold to Compute freshness of all attributes was kept under 2 and the value was within the attribute range and hence the error rate coincides. The results show how the effect of data quality of sensors affects the error rate hence the reasoning process. During the simulation, we altered the 
sensor values from the inner to outer regions and the freshness value between 2 and 3 . We used a freshness threshold values $>2$. The graph $X$ axis represents the simulation runs and the $\mathrm{Y}$-axis the error rate accuracy. We compute the error rate accuracy by changing sensor freshness and error probability values and comparing actual situation occurrence to the one suggested by R-CS and CS. The results show that incorporating data freshness heuristics greats improves the reasoning accuracy.

\subsection{Situation Partitioning and Re-Computing Weights}

We computed the confidence using R-CS algorithms presented in figure 2 and 3 and compare it with CS approach in this section. We simulated the system based on different sets of attributes values and partitioners. Table 3 gives the universal situation definitions with the partitioning parameter. The temporal situation space definition computed during simulation considers the first partition before expanding it search into other partitions.

\begin{tabular}{|c|c|c|c|c|c|}
\hline Partitioner & $0<\delta<=2$ & & $2<\delta<=5$ & & \\
\hline $\begin{array}{l}\text { Situation: } \\
\text { Attributes }\end{array}$ & $\begin{array}{l}\text { Presentation } \\
\text { Regions }\end{array}$ & & & $\begin{array}{l}\text { Relevance } \\
\text { (0 to } 5)\end{array}$ & Partition \\
\hline A1 & "= DIM" & "! ON" & "! OFF" & 3 & P1 \\
\hline A2 & " > 2 " & " <= 4" & & 3 & P1 \\
\hline A3 & "= ON" & "! OFF" & & 2 & P2 \\
\hline A4 & $\begin{array}{l}">=2 " \\
"=\end{array}$ & "< 8 " & & 2 & P2 \\
\hline & Presentation & & & & \\
\hline A5 & Mode" & "= Standby" & "! OFF" & 0.8 & P2 \\
\hline A6 & "= Inside" & "! Outside" & & 0.9 & $\mathrm{P} 2$ \\
\hline Situation: & Meeting & & & Relevance & Partition \\
\hline Attributes & Regions & & & (0 to 5$)$ & \\
\hline A1 & "! DIM" & "= ON" & "= OFF" & 3 & P1 \\
\hline A2 & "> 4 " & $"<10 "$ & & 3 & P1 \\
\hline A3 & "!ON" & "= OFF" & & 2 & $\mathrm{P} 2$ \\
\hline \multirow[t]{3}{*}{ A4 } & " > 1 " & " <= 3" & & 2 & $\mathrm{P} 2$ \\
\hline & "! & & & & \\
\hline & Presentation & & & & \\
\hline A5 & Mode" & "= Standby" & "= OFF" & 0.8 & P2 \\
\hline A6 & "= Inside" & "! Outside" & & 0.9 & P2 \\
\hline
\end{tabular}

Table 3: Universal Situation Space Definition

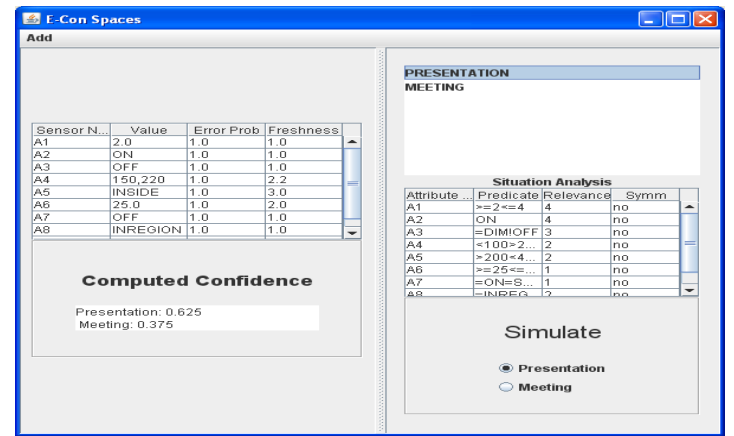

Figure 5: Simulation of Situation in E-CS
We ran simulations to compute the confidence using R-CS and CS approach using the above situation definitions by using a stream of simulated sensor data. Figure 5 is a screen shot of the simulator developed in Java and figure 6 presents the results of the simulations. As illustrated in the graph, R-CS approach results in higher 
confidence using the re-weighted algorithm compared to CS. Another key point to note is the difference in the confidence values between Meeting and Presentation situation in R-CS and CS. The difference in confidence computed for situations is greater that CS using the proposed R-CS approach which aids in increasing the confidence of the reasoning process.

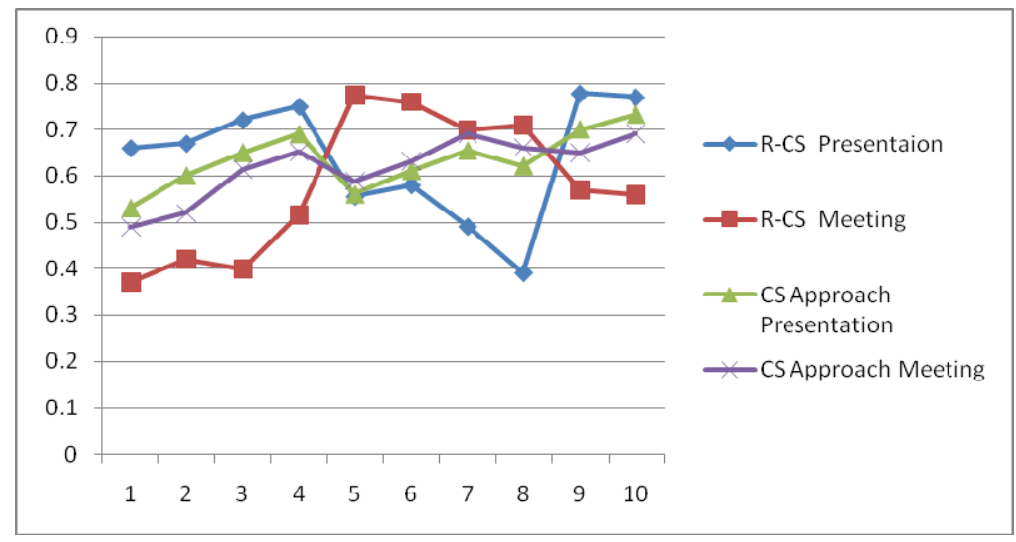

Figure 6: Confidence computed using R-CS and CS approaches

\section{Conclusion}

The paper has proposed implemented and validated R-CS, ranked dynamic situation composition based context reasoning system. We have presented the heuristics used in context spaces identifying the short falls in them. We have proposed R-CS as a solution to bridge CS shortfalls by proposing addition heuristics including partitioned universal and temporal situation spaces, sensor data quality computation and improved error estimation algorithm. The error estimation algorithm incorporated error rate and the data freshness to compute accurate contribution value which has been proved by systematic simulations and evaluations. The heuristics proposed aids the reasoning ability of the system within smart spaces. Our proposed approaches of using a dynamic set of context attributes to compute a temporal situation at time $\mathrm{T}$ (temporal situation space) and data freshness based error estimation algorithm has been validated exhaustively using simulated sensor inputs within the smart space.

R-CS has been evaluated by implementing the proposed algorithms and comparing them with the CS approach. The results show increased confidence in reasoning ability using proposed R-CS approaches over CS. The results have proved that our hypothesis and reinforces our proposal of using situation composition on the run and incorporation of heuristics like data freshness into the error estimation algorithm to improve the reasoning ability of the context aware system. We now look at further investigating approaches to identify relationships of newly discovered context attributes to existing situation spaces. 


\section{References}

[1]Context. (n.d.). The Free On-line Dictionary of Computing. Retrieved June 04, 2008, from Dictionary.com Available: http://dictionary.reference.com/browse/context

[2] Padovitz, A., Loke, S.W., and Zaslavsky, A. Towards a Theory of Context Spaces , in C Das and M Kumar (eds), Proceedings of the Second IEEE Annual Conference on Pervasive Computing and Communications Workshop, Orlando, Florida, 14 - 17 March 2004, IEEE Computer Society, USA, ISBN: 0769521061, pp 38-42.

[3] Mendel, J.M.: Fuzzy Logic Systems for Engineering: A Tutorial. Proceedings of the IEEE 83(3), 345-377 (1995)

[4] Barron FH, Barrett BE. The efficacy of SMARTER--simple multi-attribute rating technique extended to ranking. Acta Psychologica 1996;93:23-36.

[5] Butler, J., Morrice, D. J., and Mullarkey, P. W. 2001. A Multiple Attribute Utility Theory Approach to Ranking and Selection. Manage. Sci. 47, 6 (Jun. 2001), 800-816

[6]Fox, D., Hightower, J., Liao, L., Schulz, D., Borriello, G.: Bayesian filtering for location estimation. IEEE Pervasive Computing (2003)

[7] Castro, P., Munz, R.: Managing context data for smart spaces. IEEE Personal Communications 7(5), 4-46 (2000)

[8] Zadeh, L., Outline of a New Approach to the Analysis of Complex Systems, IEEE Transactions on System, Man and Cybernetics Vol. 3, 1973.

[9] Shulin Y., Chang K. C., .Modular Neural Net Architecture for Automatic Target Recognition., In Proc. of Signal Processing, Sensor Fusion, and Target Recognition Vol., 8-10, pp. 166-177, Orlando, Florida, April 1996.

[10] Buchholz T., Kpper A., and Schiffers M.. Quality of context: What it is and why we need it. In Proceedings of the Workshop of the HP OpenView University Association, Geneva, Switzerland, 2003.

[11] Salber D., Dey A. K. and Abowd G. D.. A conceptual framework and a tool-kit for supporting the rapid prototyping of context-aware applications. Anchor article of a special issue on context-aware computing in the Human-Computer Interaction (HCI) Journal, 16(24), 2001.

[12] Ranganathan A., Campbell R. H., A Middleware for Context-Aware Agents in Ubiquitous Computing Environments., In ACM/IFIP/USENIX International Middleware Conference, pp. 16-20, Rio de Janeiro, Brazil, June, 2003

[13] Chen H., Finin T., Anupam J., 2003, An Intelligent Broker for Context-Aware Systems, In Proc. of Fifth International Conference on Ubiquitous Computing (Ubicomp’03), October 2003.

[14] Padovitz, A., Loke, S.W., Zaslavsky, A., Burg, B., Bartolini, C.: An Approach to Data fusion for Context-Awareness. In: Dey, A.K., Kokinov, B., Leake, D.B., Turner, R. (eds.) CONTEXT 2005. LNCS (LNAI), vol. 3554, pp. 353-367. Springer, Heidelberg (2005)

[15] Jayaraman P.P., Zaslavsky, A., Delsing, J., Smart Sensing and Sensor Data Collection on the move for Modelling Intelligent Environments, 1st Conference on Smart Spaces ruSMART, St.Petersburg, Russia, 2008 